\title{
La validité des extrapolations aux faibles doses
}

\author{
Introduction
}

\author{
J.C. NENOT*
}

(Manuscrit reçu le 2 juin 1989)

Pour des raisons à la fois scientifiques et opérationnelles, le risque des faibles doses de rayonnements doit être quantifié avec une précision suffisante. Les besoins scientifiques concernent les disciplines comme la radiobiologie et la radiopathologie, dont les acquis servent de base à l'évaluation des risques et sont utilisés dans le domaine pratique de la protection radiologique pour fixer les contraintes indispensables au maintien d'un état satisfaisant de la santé de l'homme.

Les connaissances sur les effets des rayonnements ont suivi de peu la découverte des rayons $X$ par Roentgen en 1895 et celle de la radioactivité naturelle par Becquerel en 1896 ; c'est ainsi que la première observation de cancer radioinduit chez l'homme a eu lieu en 1902, suivie rapidement de l'observation animale en 1910 par Pierre Marie. Depuis cette époque, les études se sont multipliées et les problèmes qui se posent ne sont pas dus à un manque de données ou à leur insuffisance, mais se situent au niveau de l'interprétation. Les données de base dont on dispose proviennent soit de l'expérimentation animale soit de l'observation de groupes humains particulièrement exposés aux rayonnements.

L'expérimentation animale, poursuivie activement depuis une quarantaine d'années, a porté sur les effets cancérigènes ; pour ces derniers, elle constitue la seule source d'information quantitative. Elle a utilisé de nombreuses espèces animales, qu'il s'agisse de rongeurs, d'ovins, de porcins ou de primates. Comme il est toujours difficile et risqué d'extrapoler de l'animal à l'homme, les avantages de l'expérimentation animale ne concernent pas la quantification du risque; les enseignements qui en ont été tírés sur les mécanismes d'induction des effets et sur les lois générales d'ordre qualitatif sont irremplaçables. L'expérimentation animale présente, en outre, de nombreux autres avantages : le chercheur maîtrise les conditions physiques de l'exposition et peut jouer sur le facteur temps, en utilisant des espèces dont la durée de vie permet d'espérer l'obtention de résultats dans des délais raisonnables.

* Commissariat à l'énergie atomique, Institut de protection et de sûreté nucléaire, Département de Protection sanitaire, Services d'hyglène radiologique, $\mathrm{BP} \mathrm{n}^{\circ} 6,92265$ Fontenayaux-Roses Cedex. 
Les études épidémiologiques ont porté essentiellement sur l'apparition des cancers solides et des leucémies ; elles consistent à comparer la fréquence d'apparition d'une affection donnée parmi un groupe exposé à la fréquence naturelle. Les groupes étudiés ont été exposés pour des raisons médicales, professionnelles ou sont constitués des populations d'Hiroshima et Nagasaki. Les enquêtes qui ont suivi des groupes irradiés pour raisons médicales sont multiples et concernent de nombreux malades ; il faut spécialement citer les malades irradiés pour spondylarthrite ankylosante avec un seul traitement aux rayons $X$ entre 1935 et 1954, les enfants irradiés au cuir chevelu pour traitement des teignes, les femmes dont le bassin a été irradié pour diverses affections gynécologiques et celles qui ont subi une irradiation des seins pour affections bénignes. Les limites de ce type d'étude sont nombreuses : population spécifique, avec la difficulté de comparaison avec une population témoin, effectif limité, irradiations localisées, dosimétrie incertaine, et surtout doses élevées se situant dans une très large gamme, entre 0,1 et 10 Gy environ. Le suivi des groupes de professionnels est soumis aux mêmes écueils, qu'il s'agisse des peintres de cadrans lumineux contaminés par le radium 226 ou des mineurs d'uranium, américains, tchèques, canadiens ou français. Pour les premiers, il s'agit d'une exposition interne avec des doses reçues sur des temps très longs et, pour les seconds, d'une exposition bronchopulmonaire, à faible débit mais cumulant souvent de fortes doses, avec la particularité des irradiations alpha, dont l'efficacité biologique relative est toujours difficile à apprécier dans l'absolu.

C'est pourquoi les populations d'Hiroshima et de Nagasaki sont finalement considérées comme la référence la plus sûre : les groupes constitués en 1950, suivis très attentivement, comprennent près de 300000 personnes. En fait, la taille du groupe conditionne la précision du facteur de risque que l'on veut évaluer. Ainsi, on peut, connaissant l'ordre de grandeur du risque et la différence entre les doses reçues par le groupe exposé et le groupe témoin, évaluer la taille minimale et le temps d'observation nécessaires pour apprécier un excès significatif de cancers. II est évident que plus la différence est petite, plus grand devra être l'effectif étudié et long le temps d'observation. D'ailleurs, les résultats des études prospectives ne peuvent être complets que lors de l'extinction complète de la cohorte. En d'autres termes, il existera toujours une butée, certes de plus en plus basse au fur et à mesure que les effectifs sont plus importants, mais cette butée constitue la limite pratique des études épidémiologiques telles qu'elles sont actuellement conçues. Pour les survivants d'Hiroshima et Nagasaki, se surajoutent des problèmes quasi insurmontables dès que l'on souhaite quantifier le risque aux faibles doses et aux faibles débits : exposition reçue instantanément, population non représentative du Japon de l'époque, donc encore moins de l'individu standard européen actuel. Les dernières révisions dosimétriques, qui ont réévalué non seulement les kermas mais aussi les doses absorbées en reprenant scrupuleusement chaque dossier individuel, ne permettent pas de résoudre la question de l'atténuation du risque en fonction de la diminution du débit de dose. II est cer- 
tain que le risque augmente avec la dose, qu'une relation linéaire (incrément de risque constant pour un incrément donné de dose quel que soit le niveau de cette dose) est vraisemblable pour certains types de cancers et que le temps de latence est, avant tout, dépendant du type de cancer. La gamme de radiosensibilité des organes et tissus est très grande et ce n'est qu'après une trentaine d'années que certains cancers sont apparus indiscutablement en excès chez les survivants japonais.

Les deux grandes questions : (1) quel est le risque cancérogène en rapport avec une exposition aiguë ? et (2) quel est le facteur de correction à utiliser pour rendre compte de l'étalement de l'exposition dans le temps et quelle est son expression ? sont loin d'être résolues par ce type d'études. La première question obtient des réponses depuis plusieurs décennies, avec une tendance évolutive vers un accroissement du risque. La raison en est sans doute double : (1) le choix du modèle de projection parmi tous ceux proposés est difficile et ceci incite les biologistes à ne pas exclure celui qui donne les résultats les plus pessimistes, et (2) les cohortes étudiées n'ont pas encore entièrement disparu. Les survivants devenant de plus en plus vieux présentent de plus en plus de cancers, et de nouveaux cancers d'organes jusqu'à présent indemmes apparaissent. La deuxième question est beaucoup plus difficile, alors qu'elle conditionne de très nombreux problèmes, fondamentaux et opérationnels. Les effets des faibles doses sont-ils compensés ou annulés par les phénomènes de réparation et, de ce fait, en plus d'une relation curviligne à ce niveau de dose, faut-il considérer un seuil là où il n'y en a pas pour les débits élevés ? Comment définir et justifier l'utilisation d'un facteur d'atténuation valable pour la pratique courante, donc de valeur unique ? Le Comité scientifique des Nations unies pour l'étude des effets des rayonnements ionisants (UNSCEAR) a indiqué dans son rapport publié en décembre 1988 que ce facteur se situait entre 2 et 10 . Pour mémoire, les valeurs recommandées du facteur de réduction pour les faibles doses ont été les suivantes,:

$$
\begin{array}{llr}
\text { CIPR (1977) } & : & 2 \\
\text { UNSCEAR (1986) } & :>5 \\
\text { UNSCEAR (1988) } & : 2-10
\end{array}
$$

en définissant les faibles doses et les faibles débits par les niveaux de 0,2 Gy et 0,05 mGy.min $\mathrm{m}^{-1}$.

Les vrais problèmes demeurent donc entiers. II est regrettable que des implications non scientifiques viennent de temps à autre fausser le débat et lui ôter quelque peu de la sérénité dont il ne saurait se passer. II faut espérer qu'à défaut de la lumière totale, des discussions saines portant sur l'ensemble des résultats et les prenant tous en compte donneront un éclairage réaliste du risque en rapport avec de faibles doses de rayonnements. 